\title{
Procedimiento para el diseño de un producto turístico integrado en Cuba
}

DOI: $10.22403 /$ UROOMX/TYP04/10

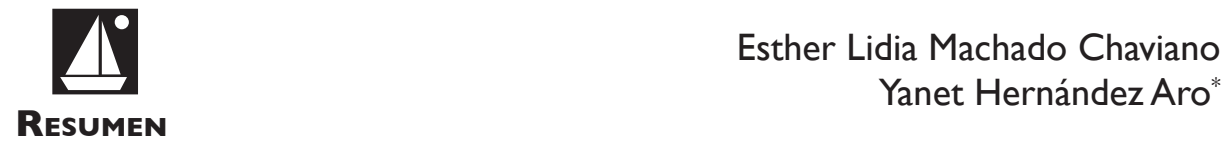

Este trabajo aborda el diseño de un procedimiento participativo para la creación de productos turísticos dirigidos a mejorar el marketing de Cuba y se aplica al producto nombrado Ruta Guerrillera. La investigación se basó en el análisis de metodologías para el desarrollo de nuevos productos a partir de las nuevas tendencias del mercado y de su repercusión en la evolución del marketing relacional al marketing inverso. Como resultado se ofrece un modelo que integra los atractivos, los componentes del destino y su autenticidad con las nuevas tendencias del mercado internacional para elevar el desarrollo turístico de Cuba.

Palabras | Cuba, Ruta Guerrillera, marketing, productos turísticos.

CLAVE

*Universidad Central Marta Abreu de Las Villas / esthermc@fce.uclv.edu.cu / yanetha@fce.uclv.edu.cu 


\section{Introducción}

En la sociedad contemporánea surgen nuevas formas de pensar que buscan mayor racionalidad, lo que propicia la construcción de un nuevo proyecto de futuro autogenerador y multirrelacional.

Las tendencias señalan las líneas de evolución que se van a confirmar en el futuro y se caracterizan por la internacionalización de los mercados; la democracia directa, participativa y multiforme; la supercompetitividad de productos de bajo precio y máxima calidad; la supertendencia, que incluye en un mismo producto múltiples servicios y valores; las tecnologías multimedias interactivas; las estrategias conjuntas interempresas; la visión del cuidado y conservación del medioambiente; el conocimiento y el talento; la flexibilidad y la creatividad; la diferenciación y la innovación e inventiva continua (Gianoten, I99I). Se imponen, por tanto, las leyes del mercado como única opción para alcanzar el desarrollo y la evolución del marketing que trata de dirigir y controlar al cliente, a un nuevo enfoque que busca conectar y colaborar con el cliente, el llamado marketing colaborativo o marketing inverso.

Los principios del nuevo marketing centran sus esfuerzos en lograr la transición del enfrentamiento a la colaboración para crear un estrecho vínculo que determinará el éxito o no de la empresa: entre ésta, los clientes y los colaboradores externos (Kotler, 2005); esto implica que los procedimientos tradicionales para el diseño de productos deban evolucionar con el tiempo actual en que la inmediatez de la información y la segmentación total han provocado cambios en los hábitos de compra de los consumidores.

Se hace necesario entonces investigar desde la perspectiva del nuevo marketing los factores determinantes en los procedimientos para el diseño de productos y más específicamente de productos turísticos, cuya intangibilidad, inseparabilidad de la producción y el consumo, variabilidad, estacionalidad e interdependencia inciden negativamente en el diseño.

\section{Desarrollo}

Todo diseño de productos turísticos debe ser considerado como un trabajo artístico, original, con los siguientes objetivos: rentabilidad económica, social y ambiental, y la satisfacción de la demanda. Definimos el producto turístico como la combinación de una serie de elementos tangibles e intangibles que 
sólo se reconocen al momento mismo del consumo. Los especialistas del marketing, Santesmases (1992), Mazur (2000) con el Despliegue de la función de calidad (QFD por sus siglas en inglés), Kotler (1992) y Martín (2003), han planteado procedimientos adaptables al diseño de productos turísticos.

\section{Análisis comparativo de los procedimientos estudiados}

Mediante un análisis comparativo se definieron las etapas que debe tener cualquier procedimiento para el diseño de un producto (cuadro I).

Cuadro I.Análisis comparativo de metodologías para el diseño de un producto turístico

\begin{tabular}{|c|c|c|c|c|c|c|c|c|c|c|}
\hline $\begin{array}{l}\text { Metodologías/ } \\
\text { Etapas }\end{array}$ & $A$ & B & C & $D$ & $E$ & $\mathrm{~F}$ & G & $\mathrm{H}$ & 1 & J \\
\hline Santesmases & & & $\mathrm{X}$ & $x$ & $x$ & $x$ & & $x$ & $\mathrm{X}$ & $\mathrm{x}$ \\
\hline QFD & $x$ & $x$ & $x$ & $x$ & & & $x$ & $\mathrm{X}$ & $x$ & \\
\hline Kotler & & & $x$ & $\mathrm{x}$ & $\mathrm{X}$ & $x$ & $x$ & $x$ & $x$ & $x$ \\
\hline Martín & & & $x$ & $x$ & $x$ & & $x$ & $x$ & $\mathrm{x}$ & $\mathrm{x}$ \\
\hline \multicolumn{5}{|c|}{ A: Objetivos } & \multicolumn{6}{|c|}{ F: Estrategias de marketing } \\
\hline \multicolumn{5}{|c|}{ B: Necesidades de clientes } & \multicolumn{6}{|c|}{ G:Análisis del negocio } \\
\hline \multicolumn{5}{|c|}{ C: Generación de ideas } & \multicolumn{6}{|c|}{$\mathrm{H}$ : Desarrollo del producto } \\
\hline \multicolumn{5}{|c|}{ D:Tamizado de ideas } & \multicolumn{6}{|c|}{ I: Prueba del mercado } \\
\hline \multicolumn{5}{|c|}{ E: Concepto del producto } & \multicolumn{6}{|c|}{ J: Lanzamiento al mercado } \\
\hline
\end{tabular}

Se pudo constatar que en todos los procedimientos sólo interviene el creador del producto, es decir, su futuro explotador, y no las demás empresas que ofrecen sus servicios y que también intervienen directamente en el producto, lo cual provoca que el resultado del diseño responda únicamente a modalidades muy puntuales dentro de la demanda turística, con exclusión de otras. Al detectar esta debilidad, se buscaron técnicas que permitieran en el diseño la interrelación de todos los factores que intervienen en el producto.

Debido a que en la bibliografía consultada no se encontraron elementos que pudieran satisfacer las demandas actuales de los mercados para la creación de productos turísticos integrados, se creó un procedimiento que 
establezca la participación activa de todos los factores, instituciones y empresas que involucrados en el producto.

\section{Propuesta para el diseño de productos turísticos}

El procedimiento que se propone tiene por objetivo diseñar productos turísticos integrados donde la autenticidad constituya una ventaja competitiva, es decir, los recursos históricos, culturales y naturales que podrían incorporarse al producto, sin que se pierda la esencia de la visita del cliente y lograr una interrelación con el resto de los prestatarios que intervienen en el producto.

El carácter participativo del procedimiento está dado por la intervención de todas las empresas, organizaciones políticas y populares, que interactúan con la línea de producto que se diseña, bajo la coordinación de las agencias de viajes. Esta concepción constituye una herramienta de aprendizaje que permite el redescubrimiento del saber popular, las exigencias de un conocimiento vinculado a las personas que configuran su contexto, dando la oportunidad a las poblaciones rurales y urbanas de exponer su propia situación y condiciones de vida.

Este procedimiento consta de varias etapas que establecen ciclos para cada una de las variables del marketing operativo (producto, precio, comunicación y distribución); cada uno concluye con la definición de las estrategias y acciones que se deben desarrollar.

\section{BÚSQUEDA Y ANÁLISIS DE LA INFORMACIÓN}

Análisis de la situación de las demandas del mercado y las tendencias futuras. Permite identificar cuáles son los mercados disponibles; esto es, hacia qué mercados se puede dirigir la oferta; cuáles son sus necesidades y preferencias. Con esta información es posible segmentar el mercado y profundizar en su estudio.

Análisis de la competencia. Se realiza un inventario y análisis de las principales empresas que ofertan sus productos en el segmento seleccionado, sus precios y la estrategia de posicionamiento. El análisis se hace utilizando las cinco fuerzas de Porter (2002). 
Inventario de recursos. Se elabora un inventario de atractivos dentro de la situación competitiva y de mercado, para generar un grupo de ideas. Es muy importante tener en cuenta el estado actual de los recursos, su accesibilidad y determinar lo que constituye el atractivo principal.

\section{DISEÑO DEL PRODUCTO}

Concepción de nuevas ideas y su selección. Supone una búsqueda sistemática de nuevas ideas para generar productos turísticos. Las ideas deben estar estructuradas de manera que incluyan las preferencias, gustos y tendencias del mercado, además de la infraestructura existente. Para generar ideas pueden utilizarse como fuentes:

- Clientes actuales: los propios clientes de la empresa, que conocen y utilizan sus productos, pueden proporcionar sugerencias sobre nuevos productos o mejoras en los actuales.

- Empleados: también los empleados de la empresa, y en especial los vendedores, que tienen un contacto directo con el mercado, pueden aportar ideas para desarrollar nuevos productos.

- Distribuidores y proveedores: los distribuidores están más próximos al mercado y tienen un mejor conocimiento de las necesidades y problemas de los consumidores, por lo que pueden proporcionar sugerencias para nuevos productos.

- Científicos e inventores: la empresa puede disponer de un equipo investigador propio o establecer convenios con la universidad u otros centros de investigación para desarrollar nuevos materiales, fórmulas o aplicaciones.

- Competidores: los productos lanzados por los competidores pueden estimular el desarrollo de otros similares o mejores.

Métodos para obtener nuevas ideas

- Brainstorming. Consiste en una reunión de personas (menos de 15) de todo tipo, en la que cada una va exponiendo ideas sobre el tema de análisis, de forma espontánea y desinhibida; las ideas no son criticadas por los asistentes. 
- Cinética. Puede ser considerada como una sesión de brainstorming más estructurada, en la que mediante un cuidadoso examen de la definición del problema se especula sobre soluciones posibles.

Cribado de ideas. El cribado de ideas supone la eliminación de las que son inviables o pobres. El producto, cuya idea se evalúa, debe ser compatible con la imagen y los objetivos de la empresa, la cual debe evaluar la tecnología requerida por el producto y determinar si concuerda con su experiencia y capacidad técnica; del mismo modo debe considerar si cuenta con la capacidad legal de llevar a término la idea. Los recursos de la empresa (capacidad de producción, de investigación y desarrollo, almacenaje y recursos financieros), así como los canales de distribución necesarios son factores que condicionaran también la aceptación o rechazo de la idea de un nuevo producto.

Definiendo los atributos y concepto del nuevo producto. Determinar el concepto del producto partiendo de los atributos diferenciadores que deseamos que tenga, será lo que constituya la base del producto, su raíz, lo cual nos permitirá adaptarlo a las características de cada mercado, sin perder la esencia del producto.

El concepto del producto consiste en su descripción detallada, en términos que tengan significado para el consumidor.

El concepto de producto nuevo debe estar determinado por el punto de vista del comprador; es decir, en cómo lo percibe. Para que el producto nuevo sea aceptado en el mercado, debe presentar alguna diferencia significativa con respecto a los existentes; aportar alguna nueva idea no experimentada o ventaja de precio o rendimiento.

Despliegue de clientes y sus requerimientos. De acuerdo con la idea desarrollada se definen los posibles segmentos de mercado y sus requerimientos básicos.

Análisis de los requerimientos y los atributos. Con los elementos obtenidos en los dos pasos anteriores se confecciona una matriz donde se analizan los atributos del producto en relación con los requerimientos básicos de los segmentos seleccionados para su diseño. Se establece si el concepto del producto corresponde a los requerimientos del mercado. 
Diseño del producto. Define el nombre, itinerario, los servicios que se brindaran y los procesos del producto.

Definición de Estrategias. Una vez diseñado el producto, debe desarrollarse una estrategia comercial.

\section{DEFINICIÓN DEL PRECIO}

Cotizaciones. Se realiza un inventario de los prestatarios del producto, los servicios que ofrecen con sus respectivos presupuestos. Con esta información se elabora una ficha de costos.

Fijación del precio. Obtenido el costo del producto se establecen los índices de beneficios que fija la agencia de viajes, se estudian los precios de productos similares, y se define el precio del propio producto por segmentos de mercado.

Definición de estrategias. Debe desarrollarse una estrategia comercial para el precio, teniendo en cuenta las fases del ciclo de vida del producto y las adecuaciones que debe tener en función del segmento de mercado al que esté dirigido.

\section{POSICIONAMIENTO}

Definición de la estrategia de posicionamiento. Permite planificar el proceso de inserción del nuevo producto en el mercado, mediante las estrategias y acciones a desarrollar en el mix de comunicación.

\section{DefinICIÓN DEL CANAL DE DISTRIBUCIÓN}

Selección del canal. En una primera etapa se analizarán los touroperadores y agencias de viajes que en el país con representaciones operan los segmentos de mercados seleccionados. Luego se realizará en cada mercado el estudio y levantamiento de los principales touroperadores que trabajan estos segmentos, para elaborar un listado de los touroperadores de mayor presencia en cada uno de los mercados. 
Procedimiento para el diseño

de un producto turístico

\section{RetROALIMENTACIÓN}

Se diseñará un sistema de encuesta del que se obtenga información sobre el grado de satisfacción del cliente y las principales sugerencias, de forma tal que el producto se encuentre permanentemente en retroalimentación.

El desarrollo del procedimiento que se propone puede apreciarse en el siguiente diagrama.

Procedimiento para el diseño de un producto turístico integrado

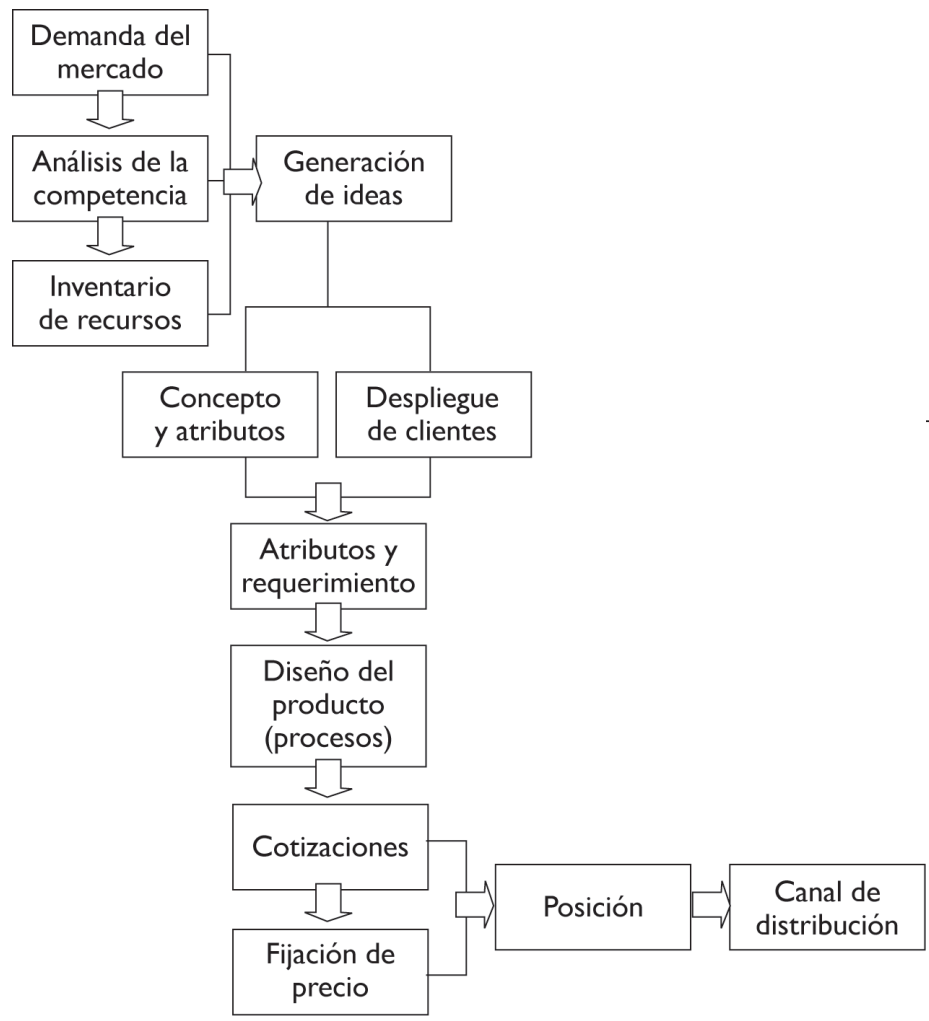

Fuente: Elaborado por los autores. 


\section{Diseño de un producto turístico integrado: Ruta Guerrillera}

Para el diseño de este producto se siguieron los pasos planteados en el procedimiento propuesto.

Se creó un equipo de trabajo, rectorado por la agencia de viajes receptora Cubanacán, y las empresas propietarias y administradoras de los principales recursos naturales y artificiales (Empresa de Flora y Fauna, Empresa de Ciencia e Innovación Tecnológica y Medio Ambiente, Patrimonio y Sectorial de Cultura) y organizaciones políticas y populares que interactúan con la línea de producto que se quiere diseñar, cumpliendo con el carácter participativo y multicriterio del procedimiento propuesto.

\section{BÚSQUEDA Y ANÁLISIS DE LA INFORMACIÓN}

Demandas y tendencias del mercado. Los cambios producidos en la actividad turística dan lugar a la aparición de nuevas tendencias que reemplazarán los destinos y actividades propias del turismo de masas por otras que se adapten mejor a las nuevas características de los turistas. A esta tendencia se le conoce como turismo alternativo, el cual exige nuevas formas de hacer turismo en las que básicamente se eleve la calidad de los servicios, propicie la participación del turista en el diseño y en las actividades que realiza, y una mayor sensibilidad hacia las cuestiones medioambientales.

Aprovechando las tendencias de la sociedad de entrar en contacto directo con los espacios naturales, se estudiaron las principales demandas insatisfechas que caracterizan al mercado europeo según estudios de la Organización Mundial del Turismo: viajes de descubrimiento (visitas a espacios naturales, culturas y tradiciones autóctonas), senderismo, cicloturismo, observación de la flora y la fauna, aventuras y diversión en la naturaleza, actividades al aire libre, patrimonio natural y cultural, y visitas a áreas naturales protegidas.

Inventario de recursos. Se hizo en varias etapas: primero se dividió el país en regiones naturales desde el punto de vista geográfico, se inventariaron todos sus recursos para luego agruparlos por tipo: flora, fauna, paisajismo, espeleología y niveles de complejidad; en una segunda etapa se trabajó con los recursos históricos, culturales y comunitarios; en tercer lugar se realizó un 
levantamiento de toda la infraestructura turística y complementaria. Con los elementos anteriores se identificaron los puntos comunes entre cada región con el objetivo de conectarlas entre sí, encontramos que los valores históricos del proceso revolucionario cubano se desarrollaron en tres de los escenarios naturales (región sur oriental, centro oriental y central). De ahí surgió la idea de crear una ruta que tuviera como eje temático el proceso revolucionario y se le denominara Ruta Guerrillera.

Definición de atributos y conceptos del nuevo producto

Atributos

- Fuerte componente histórico como atributo básico del producto, el cual debe contar con el reconocimiento a nivel nacional e internacional.

- A partir de esquemas generales, se establecerán las combinaciones adecuadas a los requerimientos de cada segmento.

- Se deberá aprovechar la infraestructura existente que posibilitará el montaje de una base de operaciones flexible y móvil, que permita reducir los costos y garantizar el uso de rutas y recursos no convencionales, en aras de garantizar la competitividad del producto.

- La definición estratégica del producto se hará desde la primera fase con la integración de los distintos factores y organizaciones políticas y de masas.

- Desarrollará servicios no convencionales que permitan la integración y participación de la mayoría de los grupos empresariales registrados en el Ministerio de Turismo.

- Utilizará la modalidad de aventura como forma de presentación y penetración de este producto en los diferentes mercados.

- Potenciará la participación y la interactividad de los clientes con el producto, en escenarios previamente seleccionados.

- Potenciará el intercambio y vida en comunidades rurales.

- Contribuirá a la preservación, desarrollo y promoción del patrimonio histórico y natural cubano. 


\section{Diseño del producto Ruta Guerrillera}

Concepto del producto. Recrear una ruta temática de personalidades, ambientes, hechos y escenarios históricos-naturales con un alto reconocimiento internacional; en la modalidad de aventura, de modo tal que las experiencias del grupo de visitantes y el intercambio con la comunidad contribuyan a generar una imagen de autenticidad, cubanía y sostenibilidad del producto.

Despliegue de clientes. Se definieron los segmentos de mercados a los cuales podría ser lanzado el producto en función de las motivaciones de viajes. En el análisis de los mercados se detectó una demanda de la práctica del turismo de naturaleza no sólo en ecoturistas, sino también en el segmento estudiantil; por las diferencias de las necesidades de ambos segmentos se decidió trabajarlos por separado. Se identificaron, entonces, los requerimientos para cada segmento de mercado y sus niveles de incidencia, y fueron seleccionados aquellos que ofrecían mayores ventajas de comercialización para el destino Cuba.

Ubicación, clasificación y selección de los atributos. Partiendo del inventario que se realizó por regiones, se seleccionaron las provincias y locaciones que cumplían con los atributos para la confección del producto: Granma, Sancti Spíritus y Villa Clara; y las locaciones, por citar algunas: Las Cloradas, Alegría de Pío, La Plata, Caballete de Casa, Gavilanes, Pedrero, Loma del Capiro, Universidad de Las Villas, Tren Blindado y Memorial. Luego se elaboraron los programas para cada segmento de mercado y se trazaron la ruta histórica y la ruta de las actividades.

Matriz atributos/requerimientos. El siguiente paso implicaba establecer la relación que debía existir entre los atributos de los programas y los requerimientos de los diferentes segmentos.

Construcción del proceso de la Ruta. Se definieron los procesos por los que se rige la Ruta, lo cual permitió establecer el tiempo real de interacción de cada uno de los elementos que involucra el producto $y$, por ende, la identificación de todos los factores que intervienen, así como los recursos que se necesitan. Es por esto que el proceso de la Ruta se construyó para cada uno de los posibles segmentos a los que va dirigido el producto. 
Procedimiento para el diseño

de un producto turístico

\section{DEFINICIÓN DEL PRECIO}

Se elaboró la ficha de costo del producto, siguiendo la estrategia de fijación de precios a partir del análisis de la competencia.

\section{Posicionamiento}

Se analizó el triángulo estratégico para identificar las ventajas competitivas del producto y utilizarlas como elemento básico de su posicionamiento; se diseñaron el logotipo y las acciones de merchandising, y se definieron los medios para su inserción en cada uno de los mercados.

\section{CANAL DE Distribución}

Por último, se escogieron los canales de distribución. La tarea se dividió en dos momentos, primero se realizó un listado de las agencias de viajes y turoperadores que operaban en la isla con los mercados seleccionados; luego, los que trabajaban con productos similares. En un segundo momento se localizaron turoperadores que trabajaban con productos de naturaleza, pero que no tenían contratos con Cuba; esto último se hizo a través del Ministerio de Turismo.

\section{Conclusiones}

Las nuevas exigencias del mercado hacen necesario el diseño de productos integrados que consideren recursos tanto históricos y culturales como naturales, donde la autenticidad constituya una ventaja competitiva y además el logro de una interrelación con el resto de los prestatarios que intervienen en el producto.

La inserción de la participación en los procedimientos actuales aparece como el redescubrimiento del saber popular, las exigencias de un conocimiento ligado a las personas que configuran su contexto.

En la revisión bibliográfica se han encontrado diversos procedimientos como los de Santesmases, QFD, Kotler y Martín, sin embargo, ninguno responde al diseño de productos turísticos integrados.

Teniendo en cuenta lo anterior se elaboró un procedimiento participativo que permite la intervención de todos los factores involucrados directa e indirectamente en el producto. Se analizaron los objetivos de cada una de 
las instituciones implicadas, la situación del mercado, la concepción de nuevas ideas y su selección, hasta llegar a la creación y lanzamiento del producto y la necesaria y constante retroalimentación.

\section{FUENTES CONSULTADAS}

Cárdenas Tabares, Fabio (1995). Producto turístico. México:Trillas.

Chan, Nelida (1994). Circuitos turísticos, programación y cotización. Buenos Aires: Librerías Turísticas.

Colina, J. M. "Marketing turístico", [en línea]. Disponible en: http://www. monografias.com. 04/04/06.

Gianoten, V. y T. De Wit, (199I). “Un caso de autoorganización popular”, en Fals Borda et al.Acción y conocimiento. Como romper el monopolio con investigación-acción participativa, Santafé de Bogotá, Cinep.

Kotler, Philip (1992). Dirección de marketing / F. Kotler (7 ed.). Madrid: Prentice Hall.

Lambin, Jean-Jacques (1997). Marketing estratégico. Madrid: McGraw Hill.

Martín Fernández, Ramón (2003). Fundamentos del turismo. EAEHT.

Mazur, Glenn (2000). Comprehensive Quality Function Deployment Overview. Estados Unidos: Network.

Peñarroya, Montserrat (s.f) “Los 10 principios del nuevo marketing”, [en línea]. Disponible en: http://www.daemonquest.com

Porter, Michael (2002). Estrategia competitiva: técnicas para el análisis de los sectores industriales y de la competencia, México: CECSA, $384 \mathrm{p}$.

Santesmases, M. (1993). Marketing: conceptos y estrategias ( $\mathrm{a}^{\mathrm{a}}$ ed.). Madrid: Pirámide.

Serra, Antoni (2003). Marketing turístico. Madrid: Pirámide. 
